\title{
Josephson current carried by Andreev levels in superconducting quantum point contacts
}

\author{
Akira Furusaki \\ Yukawa Institute for Theoretical Physics, Kyoto University, Kyoto 606-8502, Japan* \\ and Department of Physics, Stanford University, Stanford, California 94305-4060
}

(February 1, 2008)

\begin{abstract}
The dc Josephson effect in a superconducting quantum point contact, where supercurrent flows through a small number of channels, is reviewed. The central role of Andreev levels is emphasized which carry the whole supercurrent in short symmetric Josephson junctions including tunnel junctions. A simple intuitive view of the dc Josephson effect in a quantum point contact is given in terms of multiple Andreev reflections. The quantization of the critical current in superconducting quantum point contacts is briefly discussed.
\end{abstract}

\section{INTRODUCTION}

Recent surprising progress in microfabrication techniques has enabled us to make tiny Josephson junctions with artificial geometry. This opens a door to explore various mesoscopic effects in the transport properties of small superconducting junctions. In this paper we review the dc Josephson effect in a superconducting quantum point contact [1,2], a Josephson junction consisting of two superconductors coupled through a few quantum channels (Fig. 1). Such a superconducting quantum point contact can be created in a superconductor-2DEG-superconductor junction (2DEG: two-dimensional electron gas) [3] as well as in a mechanically controllable break junction 何. Main focus will be on current-carrying bound states that appear near a quantum point contact. Our discussion is based on the Bogoliubov-de Gennes equation, which is a two-component Schrödinger equation describing electron-like and hole-like quasiparticle excitations in superconducting systems. We show that the Bogoliubov-de Gennes equation has solutions describing bound states that are localized around the junction and decay exponentially into the superconductors. These bound states play a central role in the dc Josephson effect. The physical picture that emerges out of the solution is that the supercurrent flows as a result of multiple Andreev reflections 5 that transform an electron into a hole or vice versa. The bound states represent standing waves of electron and hole waves going back and forth between two superconductors. This view might look slightly different from a traditional picture of the dc Josephson effect explained in textbooks, according to which the supercurrent, calculated in lowest order in the tunneling Hamiltonian, flows as a result of tunneling of Cooper pairs. In fact, these views are simply two sides of a same coin.

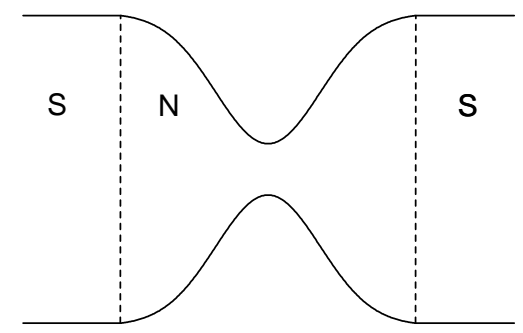

FIG. 1. Superconducting quantum point contact. The supercurrent flows through a narrow constriction in the normal region.

The importance of the bound states caused by the multiple Andreev reflections was first recognized in the early 1970s in the studies of the dc Josephson effect in long superconductor-normal metal-superconductor junctions [6 9 ]. Until the 1990s, however, it was not fully appreciated that the presence of current-carrying bound states is a general feature in any symmetric Josephson junctions including weak links, superconducting quantum point contacts, and conventional tunnel junctions. The bound states in a short junction or a point contact, whose energy depends on the phase difference of the superconductors, were explicitly constructed by solving the Bogoliubov-de Gennes equation in [10,2]. It is a curious fact that almost no attention was paid to the bound states in earlier studies of short superconducting weak links 11 13], in which the bound states show up silently as poles of the Green's function on the real axis. In fact, the Andreev bound states play a central role for various situations in mesoscopic superconducting systems. 
In this paper we will concentrate on the dc Josephson effect of $s$-wave superconductors. When a finite voltage is applied across the junction, the loop of the multiple Andreev scattering process is no longer closed, and the solutions of the Bogoliubov-de Gennes equation contain infinitely many terms each representing Andreev-reflected electron wave or hole wave 14 21]. For sufficiently small applied voltage, however, the current-voltage characteristics can be described in terms of the quasi-stationary bound states whose energy is changing slowly with time 20]. We will not discuss further the ac Josephson effect and the current-voltage characteristics of superconducting quantum point contacts in this paper.

This paper is organized as follows. In Sec. II we introduce our basic equation, the Bogoliubov-de Gennes equation, and a continuity equation for electric charge. We solve a simplified model of a Josephson junction in Sec. III to illustrate the basic idea of the supercurrent carried by Andreev bound states. We point out that a midgap surface state in a $d$-wave superconductor is essentially the same as the bound state in a short Josephson junction. We then explain a scattering approach to calculate the dc Josephson current in Sec. IV.

\section{BOGOLIUBOV-DE GENNES EQUATION}

We begin with the Bogoliubov-de Gennes equation 22

$$
\left(\begin{array}{cc}
-\frac{\hbar^{2}}{2 m} \frac{\partial^{2}}{\partial \boldsymbol{r}^{2}}+U(\boldsymbol{r})-\mu & \Delta(\boldsymbol{r}) \\
\Delta^{*}(\boldsymbol{r}) & \frac{\hbar^{2}}{2 m} \frac{\partial^{2}}{\partial \boldsymbol{r}^{2}}-U(\boldsymbol{r})+\mu
\end{array}\right)\left(\begin{array}{l}
u_{n}(\boldsymbol{r}) \\
v_{n}(\boldsymbol{r})
\end{array}\right)=E_{n}\left(\begin{array}{l}
u_{n}(\boldsymbol{r}) \\
v_{n}(\boldsymbol{r})
\end{array}\right) .
$$

where $U(\boldsymbol{r})$ represents both confining potential and scattering potential at interfaces of a superconductor and a normal metal, and $\mu$ is the chemical potential. This is the basic equation for mesoscopic superconducting systems. The upper component $u(\boldsymbol{r})$ corresponds to electrons and the lower one $v(\boldsymbol{r})$ represents holes. In principle the pair potential $\Delta(\boldsymbol{r})$ is determined from the selfconsistency condition or the gap equation, $\Delta(\boldsymbol{r})=\lambda\left\langle\Psi_{\uparrow}(\boldsymbol{r}) \Psi_{\downarrow}(\boldsymbol{r})\right\rangle$, where $\lambda(>0)$ is a coupling constant of electron-electron attraction, and \langle\rangle is an average in the equilibrium state. The field operator $\Psi_{\sigma}$ of electrons with spin $\sigma$ can be expanded with the solutions of the Bogoliubov-de Gennes equation:

$$
\left(\begin{array}{c}
\Psi_{\uparrow}(\boldsymbol{r}, t) \\
\Psi_{\downarrow}^{\dagger}(\boldsymbol{r}, t)
\end{array}\right)=\sum_{E_{n}>0}\left[a_{n \uparrow} e^{-i E_{n} t}\left(\begin{array}{c}
u_{n}(\boldsymbol{r}) \\
v_{n}(\boldsymbol{r})
\end{array}\right)+a_{n \downarrow}^{\dagger} e^{i E_{n} t}\left(\begin{array}{c}
-v_{n}^{*}(\boldsymbol{r}) \\
u_{n}^{*}(\boldsymbol{r})
\end{array}\right)\right],
$$

where the creation and annihilation operators $a_{n \sigma}$ and $a_{n \sigma}^{\dagger}$ satisfy $\left\{a_{n \sigma}, a_{n^{\prime} \sigma^{\prime}}^{\dagger}\right\}=\delta_{n, n^{\prime}} \delta_{\sigma, \sigma^{\prime}}$ and $\left\{a_{n \sigma}, a_{n^{\prime} \sigma^{\prime}}\right\}=0$. Note that $\left(-v_{n}^{*} u_{n}^{*}\right)^{\mathrm{t}}$ is an eigenfunction with energy $-E_{n}$ if $\left(u_{n} v_{n}\right)^{\mathrm{t}}$ satisfies Eq. (11). That is why we sum over the positive energy states only and introduce the second term in Eq. (2).

Following Ref. [23], we introduce the equation of continuity of electric charge, which are satisfied by the solutions of the Bogoliubov-de Gennes equation. We define

$$
\begin{aligned}
P_{e} & =-e\left[\Psi_{\uparrow}^{\dagger}(\boldsymbol{r}, t) \Psi_{\uparrow}(\boldsymbol{r}, t)+\Psi_{\downarrow}^{\dagger}(\boldsymbol{r}, t) \Psi_{\downarrow}(\boldsymbol{r}, t)\right], \\
\boldsymbol{J}_{e} & =-\frac{e \hbar}{m} \operatorname{Im}\left[\Psi_{\uparrow}^{\dagger}(\boldsymbol{r}, t) \frac{\partial}{\partial \boldsymbol{r}} \Psi_{\uparrow}(\boldsymbol{r}, t)+\Psi_{\downarrow}^{\dagger}(\boldsymbol{r}, t) \frac{\partial}{\partial \boldsymbol{r}} \Psi_{\downarrow}(\boldsymbol{r}, t)\right] .
\end{aligned}
$$

It is clear that $P_{e}$ and $J_{e}$ are electric charge density and electric current, respectively. The continuity equation for the electric charge reads $\partial_{t} P_{e}+\operatorname{div} \boldsymbol{J}_{e}+S=0$, where the source term $S$ is given by $S=(4 e / \hbar) \operatorname{Im}\left[\Delta(\boldsymbol{r}) \Psi_{\uparrow}^{\dagger}(\boldsymbol{r}, t) \Psi_{\downarrow}^{\dagger}(\boldsymbol{r}, t)\right]$. When the gap equation is satisfied, $\langle S\rangle$ vanishes, and we recover the ordinary continuity equation of the charge for the averaged quantities $\left\langle P_{e}\right\rangle$ and $\left\langle J_{e}\right\rangle$, where \langle\rangle is an average in equilibrium. When we calculate the Josephson current in a model where the pair potential satisfies the gap equation only approximately, we have to take into account the contribution from $S$ [24]. The source term can be ignored when the current is calculated inside the normal region where $\Delta(\boldsymbol{r})=0$.

\section{CURRENT-CARRYING BOUND STATES}

We shall consider a simplest model of a Josephson junction that shows the essential features of the Josephson effect. It is a one-dimensional model where the left and right superconductors have the pair potentials of the same magnitude but with different phases: 


$$
\Delta(x)= \begin{cases}\Delta_{0} e^{i \theta_{L}} & x<0 \\ \Delta_{0} e^{i \theta_{R}} & x>0\end{cases}
$$

We assume the normal region to be thin and consider an extreme case where its width is infinitesimal. In general we expect some scattering process to be present either inside the normal region or at the super-normal interfaces. We therefore introduce a scattering potential

$$
U(x)=V \delta(x) \quad V \geq 0
$$

to describe such a scattering. The model with (4) and (5) is the simplest non-trivial model for the Josephson effect. The strength of the $\delta$-function potential is characterized by a dimensionless parameter $Z \equiv m V / \hbar^{2} k_{F}$, where $k_{F}$ is the Fermi wave vector. When $Z \ll 1$ the model describes a short superconductor-normal metal-superconductor junction. In the opposite limit $Z \gg 1$, the model can be regarded as a toy model of a superconductor-insulator-superconductor tunnel junction.

We are interested in a state in the energy gap, $0<E<\Delta_{0}$. In this energy range a wave function has to decay exponentially for $|x| \rightarrow \infty$. We can write the wave function as

$$
\psi_{B}(x)=\left(\begin{array}{c}
u_{B}(x) \\
v_{B}(x)
\end{array}\right)= \begin{cases}a_{B} e^{i k_{h} x}\left(\begin{array}{c}
v_{0} e^{i \theta_{L} / 2} \\
u_{0} e^{-i \theta_{L} / 2}
\end{array}\right)+b_{B} e^{-i k_{e} x}\left(\begin{array}{c}
u_{0} e^{i \theta_{L} / 2} \\
v_{0} e^{-i \theta_{L} / 2}
\end{array}\right) & x<0, \\
c_{B} e^{i k_{e} x}\left(\begin{array}{c}
u_{0} e^{i \theta_{R} / 2} \\
v_{0} e^{-i \theta_{R} / 2}
\end{array}\right)+d_{B} e^{-i k_{h} x}\left(\begin{array}{c}
v_{0} e^{i \theta_{R} / 2} \\
u_{0} e^{-i \theta_{R} / 2}
\end{array}\right) & x>0,\end{cases}
$$

where

$$
k_{e}=\sqrt{\frac{2 m}{\hbar^{2}}(\mu+i \eta)}, \quad k_{h}=k_{e}^{*}, \quad u_{0}=\sqrt{\frac{1}{2}\left(1+i \frac{\eta}{E}\right)}, \quad v_{0}=u_{0}^{*}, \quad \eta=\sqrt{\Delta_{0}^{2}-E^{2}} .
$$

The coefficients $a_{B}, b_{B}, c_{B}$, and $d_{B}$ are determined from the matching condition at $x=0$ :

$$
\psi_{B}(-0)=\psi_{B}(+0), \quad \psi_{B}^{\prime}(+0)-\psi_{B}^{\prime}(-0)=\frac{2 m V}{\hbar^{2}} \psi_{B}(0)
$$

The eigenenergy is determined from the condition that Eq. (7) has a nontrivial solution. Within the approximation $k_{e} \approx k_{h} \approx k_{F}$ we find [10]

$$
E=E_{B} \equiv \Delta_{0} \sqrt{\frac{\cos ^{2}(\varphi / 2)+Z^{2}}{1+Z^{2}}}
$$

The solution of Eq. (7) with this energy is

$$
\begin{aligned}
& a_{B}=-N \sigma \sqrt{1+Z^{2}}\left[\sqrt{\cos ^{2}(\varphi / 2)+Z^{2}}-\sigma \cos (\varphi / 2)\right], \quad b_{B}=i \sigma N Z(1-i Z), \\
& c_{B}=N(1-i Z)\left[\sqrt{\cos ^{2}(\varphi / 2)+Z^{2}}-\sigma \cos (\varphi / 2)\right], \quad d_{B}=i N Z \sqrt{1+Z^{2}}
\end{aligned}
$$

where $\varphi=\theta_{R}-\theta_{L}, N$ is the normalization constant

$$
N^{2}=\frac{\sigma \Delta_{0} \sin (\varphi / 2)}{2 \hbar v_{F}\left(1+Z^{2}\right)^{2}\left[\sqrt{\cos ^{2}(\varphi / 2)+Z^{2}}-\sigma \cos (\varphi / 2)\right]},
$$

and $\sigma=\operatorname{sgn}(\varphi)$ for $-\pi \leq \varphi \leq \pi$. The wave function is normalized such that $\int_{-\infty}^{\infty}\left[\left|u_{B}(x)\right|^{2}+\left|v_{B}(x)\right|^{2}\right] d x=1$. Once the wave function of the positive energy state is known, the eigenstate with energy $-E_{B}$ is obtained as $\tilde{\psi}_{B}(x)=$ $\left(-v_{B}^{*}(x), u_{B}^{*}(x)\right)^{t}$. The field operator is then expanded as

$$
\left(\begin{array}{c}
\Psi_{\uparrow}(x, t) \\
\Psi_{\downarrow}^{\dagger}(x, t)
\end{array}\right)=a_{B \uparrow} e^{-i E_{B} t} \psi_{B}(x)+a_{B \downarrow}^{\dagger} e^{i E_{B} t} \tilde{\psi}_{B}(x)+\left(\text { contribution from states with }|E|>\Delta_{0}\right) .
$$

Let us assume for a while that the states above or below the energy gap $\left(|E|>\Delta_{0}\right)$ do not contribute to the supercurrent. We will see later that this is indeed the case for the model we are dealing with. We can calculate the supercurrent as the average of $J_{e}$ at $x=+0$ in the equilibrium state at temperature $T$ : 


$$
\begin{aligned}
I & =\left\langle J_{e}\right\rangle=-4 e v_{F} u_{0} v_{0}\left(\left|c_{B}\right|^{2}-\left|d_{B}\right|^{2}\right)\left[f\left(E_{B}\right)-f\left(-E_{B}\right)\right] \\
& =-\frac{e \Delta_{0} \sin \varphi}{2 \hbar\left(1+Z^{2}\right)} \sqrt{\frac{1+Z^{2}}{\cos ^{2}(\varphi / 2)+Z^{2}}} \tanh \left(\frac{E_{B}}{2 k_{B} T}\right),
\end{aligned}
$$

where $f(E)=\left[\exp \left(E / k_{B} T\right)+1\right]^{-1}$. Since the transmission probability through the $\delta$-function barrier Eq. (5) is $\mathcal{T}=1 /\left(1+Z^{2}\right)$, the normal-state conductance of this model is $G_{N}=2 e^{2} /\left[h\left(1+Z^{2}\right)\right]$. We can thus rewrite Eqs. (8) and (10) as

$$
\begin{aligned}
E_{B} & =\Delta_{0} \sqrt{1-\mathcal{T} \sin ^{2}(\varphi / 2)}, \\
I & =-G_{N} \frac{\pi \Delta_{0}}{2 e} \frac{\sin \varphi}{\sqrt{1-\mathcal{T} \sin ^{2}(\varphi / 2)}} \tanh \left(\frac{E_{B}}{2 k_{B} T}\right) .
\end{aligned}
$$

When $\mathcal{T} \ll 1$ Eq. (11b) reduces to

$$
I=-G_{N} \frac{\pi \Delta_{0}}{2 e} \sin (\varphi) \tanh \left(\frac{\Delta_{0}}{2 k_{B} T}\right)
$$

which is the well-known Ambegaokar-Baratoff formula [25]. In the opposite limit $\mathcal{T}=1$, Eq. (11b) becomes

$$
I=-G_{N} \frac{\pi \Delta_{0}}{e} \sin (\varphi / 2) \tanh \left(\frac{\Delta_{0}}{2 k_{B} T} \cos (\varphi / 2)\right)
$$

which coincides with the result obtained by Kulik and Omel'yanchuk [11] for a point contact Josephson junction. In this limit the zero-temperature Josephson current becomes maximum at $\varphi=\pi$, not at $\varphi=\pi / 2$ like in the tunnel junctions. The bound state energy is simply given by $E_{B}=\Delta_{0} \cos (\varphi / 2)$, which equals the Fermi energy $E_{B}=0$ at $\varphi=\pi$.

Equation (11b) thus interpolates between Eq. (12) of tunnel junctions with very small transmission probability and Eq. (13) of point contacts with perfect transmission. The same expression of the supercurrent interpolating the two limits has been found by many people [26, 12, 13, 27]. The phase-dependent bound state energy (8) and its implication to the Josephson effect in short junctions were emphasized in [10]. The results were then generalized to the multichannnel contacts in [28].

Equation (8) shows that there is always a bound state (Andreev level) with $E_{B} \geq 0$ unless the phase difference $\varphi$ is zero. This is true even for the tunnel junctions in which $\mathcal{T} \ll 1$ and $\Delta_{0}-E_{B} \ll \Delta_{0}$. In the above model two bound states $\left(E= \pm E_{B}\right)$ appear in the energy gap $|E|<\Delta_{0}$ but disappear when $\varphi=0$. This is a general property of short $s$-wave junctions where $\hbar v_{F} / L \gg \Delta_{0}$ with $L$ being the length of the normal region $(L=0$ in our model). In the opposite regime where $\hbar v_{F} / L \ll \Delta_{0}$, many $\left(\sim \hbar v_{F} / \Delta_{0} L\right)$ Andreev levels exist in the gap for any $\varphi$ [6]9]. These Andreev levels are easily understood as discrete levels of normal electrons confined in a quantum well of offdiagonal pair potential. They are similar to the discrete energy levels in a vortex in an $s$-wave type II superconductor [29]. A nontrivial observation we made in the above calculation is that, when $\varphi \neq 0$, two Andreev levels survive even in the limit $L \rightarrow 0$, in sharp contrast with a case where a particle is confined in a quantum well of diagonal potential barrier, in which no bound state exists in the limit $L \rightarrow 0$.

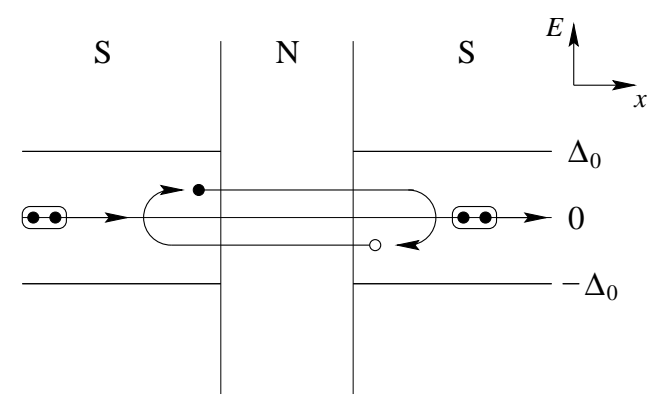

FIG. 2. Current-carrying bound state. The bound state is a manifestation of the multiple Andreev reflections and carries the whole supercurrent in a short Josephson junction. The filled (open) circle represent an electron (a hole). The ovals containing two circles are Cooper pairs. 
The bound state $\psi_{B}(x)$ is formed as a result of resonant multiple Andreev reflection process. Equation (10) shows that this state carries nonvanishing supercurrent. This can be physically understood as follows (Fig. 2). Each time an electron is Andreev reflected into a hole, a Cooper pair is effectively generated. Therefore the bound state, which represents an infinite loop of Andreev reflections (electron $\rightarrow$ hole $\rightarrow$ electron $\cdots$ ), serves as a pump that transfers Cooper pairs from one superconductor to the other. When $\mathcal{T} \approx 1$ the bound state energy has a strong dependence on the phase difference $\varphi$, which can be controlled externally by the current source. It would be very interesting if these current-carrying Andreev levels are observed experimentally using, for example, the scanning tunneling spectroscopy.

Finally we comment on midgap states that appear near the surface of a $d$-wave superconductor when the surface is not parallel to a crystal axis (Fig. (3) [30]. In fact the mechanism of the formation of the zero-energy midgap state is the same as the Andreev bound states we discussed above. As shown in Fig. 3, an electron traveling towards a surface is reflected back into the $d$-wave superconductor and is subsequently Andreev-reflected into a hole by the positive pair potential. In the next step the hole follows the same path backwards, reflected at the surface, and finally Andreev-reflected into another electron by the negative pair potential. The analogy to the Josephson junction is now obvious. The surface of the $d$-wave superconductor plays a role of the point contact with $\mathcal{T}=1$, and the sign change in the pair potential corresponds to the phase difference $\varphi=\pi$. Equation (11a) tells that there must be bound states with $E_{B}=0$ localized near the surface, which are the midgap states found in Ref. [30]. They give rise to a peak in differential conductance of a normal-insulator- $d$-wave tunnel junction [31]. The Andreev levels also play an important role in a Josephson junction made of an $s$-wave superconductor and a $d$-wave superconductor [32,33]. It is known that in such a junction there is a chance that Andreev levels appear even in the lowest-energy state, thereby leading to a ground state with broken time-reversal symmetry [34].

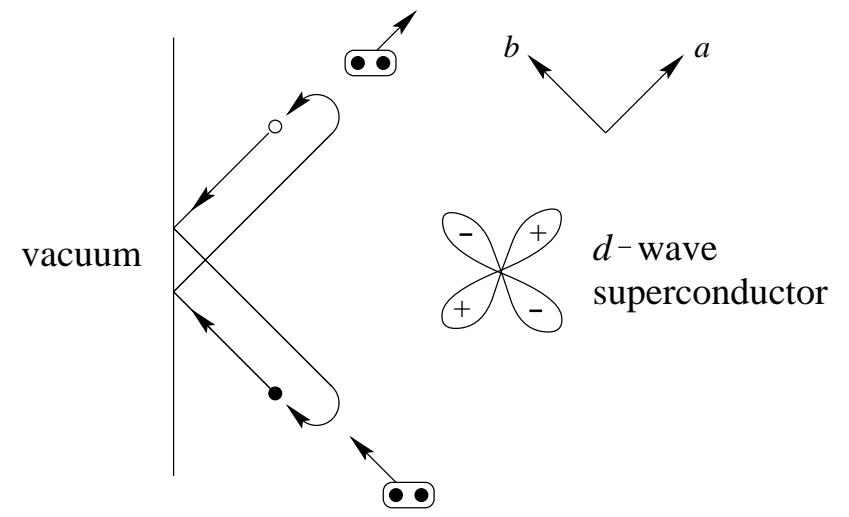

FIG. 3. Midgap state formed near the surface of a $d$-wave superconductor.

\section{JOSEPHSON CURRENT FROM SCATTERING AMPLITUDES}

We shall introduce a general expression of the dc Josephson current under the assumption that we can ignore the spatial variation of the pair potential. Here we assume

$$
\Delta(\boldsymbol{r})= \begin{cases}\Delta_{L} e^{i \theta_{L}} & x<0 \\ \Delta_{R} e^{i \theta_{R}} & x>L\end{cases}
$$

In the normal region $(0<x<L)$ the order parameter vanishes. We also assume that the potential $U(\boldsymbol{r})$ is independent of $x$ in the superconductors. We emphasize that this step-function pair potential (14) is a good approximation for mesoscopic Josephson junctions of our concern, where bulk superconductors are coupled via a few conduction channels. The use of the step-function model is justified by the following reasons. First, the presence of a point contact does not seriously affect the order parameter of bulk superconductors, because the width of the constrictions is much smaller than the superconducting coherence length $\xi$, which is the characteristic length scale for the variation of the order parameter. Second, if the normal region is made of a material different from superconducting electrodes, the contact resistance of the SN interfaces is not small due to the Fermi-velocity difference and/or due to the presence of a Schottky barrier. In this case also the pair potential is not reduced significantly near the point contact. More generally, when the resistance of the junction is much larger than those of superconductors, we may safely use the step-function model [35]. 
Since the dc Josephson current is an equilibrium current, it is given by the thermal average of $\boldsymbol{J}_{e}$. We calculate it at $x=0$ to avoid complication due to the source term $S$ :

$$
I=\left\langle\boldsymbol{e}_{x} \cdot \boldsymbol{J}_{e}\right\rangle=\left.\frac{e \hbar}{2 i m} \lim _{x \rightarrow 0} \lim _{x^{\prime} \rightarrow x} \int d y d z\left(\frac{\partial}{\partial x}-\frac{\partial}{\partial x^{\prime}}\right) \frac{1}{\beta} \sum_{\omega_{n}} \operatorname{Tr}\left[G_{\omega_{n}}\left(\boldsymbol{r}, \boldsymbol{r}^{\prime}\right)\right]\right|_{x=0},
$$

where $G_{\omega_{n}}\left(\boldsymbol{r}, \boldsymbol{r}^{\prime}\right)$ is the temperature Green's function at frequency $\omega_{n}=\pi k_{B} T(2 n+1)$ in the Nambu space $(2 \times 2$ matrix) [36]. Our assumption that the order parameter $\Delta(\boldsymbol{r})$ and the potential $U(\boldsymbol{r})$ have no dependence on $x$ in the superconductors, is strong enough that we can immediately write down $G_{\omega_{n}}\left(\boldsymbol{r}, \boldsymbol{r}^{\prime}\right)$ at $x<0$ in terms of scattering amplitudes. Its explicit expression can be found in Ref. [24]. After substituting it into Eq. (15), we obtain

$$
I=\frac{e \Delta_{L}}{\hbar \beta} \sum_{\omega_{n}} \frac{1}{\Omega_{n L}} \sum_{j}\left[a_{j j}\left(\varphi, i \omega_{n}\right)-a_{j j}\left(-\varphi, i \omega_{n}\right)\right]
$$

where $\Omega_{n L}=\sqrt{\omega_{n}^{2}+\Delta_{L}^{2}}$ and we have used the Andreev approximation where the velocities of quasiparticles are set equal to the Fermi velocity.

The quantity $a_{j k}\left(\varphi, i \omega_{n}\right)$ is a scattering amplitude for the process in which an electron-like quasiparticle of $j$ th channel traveling from the left to the junction is reflected back as a hole-like quasiparticle of $k$ th channel [24]. Equation (16) expresses the supercurrent in terms of the scattering data $a_{j j}$, just like the Landauer formula does the normalstate conductance. An important difference is that the supercurrent is proportional to the scattering amplitude, not to the probability. Equation (16) is clearly an odd function of $\varphi$, and can be expanded formally as $I=\sum_{m=1}^{\infty} I_{m} \sin (m \varphi)$. The term proportional to $\sin (m \varphi)$ corresponds to $m$ th order term in the tunneling Hamiltonian approach that is a contribution from the simultaneous tunneling of $m$ Cooper pairs. Equation (16) is thus a useful, nonperturbative formula for the dc Josephson current [37].

Let us apply it to the simple one-dimensional model we analyzed in the last section. Solving the Bogoliubov-de Gennes equation for $E>\Delta_{0}$ with potentials given by (4) and (5) gives

$$
a(\varphi, E)=-\frac{\Delta_{0}\left[E \sin ^{2}(\varphi / 2)+i \Omega \sin (\varphi / 2) \cos (\varphi / 2)\right]}{E^{2}\left(1+Z^{2}\right)-\Delta_{0}^{2}\left[\cos ^{2}(\varphi / 2)+Z^{2}\right]} .
$$

Note that the scattering amplitude has poles at $E= \pm E_{B}$. Substituting (17) with $E=i \omega_{n}$ into (16), we then find

$$
I=-\frac{e \Delta_{0}^{2}}{\hbar \beta} \sum_{\omega_{n}} \frac{\sin \varphi}{\omega_{n}^{2}\left(1+Z^{2}\right)+\Delta_{0}^{2}\left[\cos ^{2}(\varphi / 2)+Z^{2}\right]},
$$

which agrees with Eq. (10) after the frequency sum is evaluated by reducing into a contour integral. In this calculation all the contributions to the integral come from the residues at $E= \pm E_{B}$. This proves that the Andreev bound states carry the whole supercurrent in short symmetric Josephson junctions. We note that this is not true for long weak links where $\hbar v_{F} / L \ll \Delta_{0}$ [7,9] and for asymmetric junctions where $\Delta_{L}<\Delta_{R}$. Let us examine the latter case in more detail. We consider a one-dimensional model where the pair potential is given by Eq. (14) with $L=0$ and the diagonal potential is (5). A solution of the Bogoliubov-de Gennes equation for this model gives a scattering amplitude for $E>\Delta_{R}>\Delta_{L}$,

$$
a(\varphi, E)=\frac{E\left(\Delta_{R} \cos \varphi-\Delta_{L}\right)-i \Delta_{R} \Omega_{L} \sin \varphi}{E^{2}+K \Omega_{R} \Omega_{L}-\Delta_{R} \Delta_{L} \cos \varphi}
$$

where $\Omega_{R(L)}=\left(E^{2}-\Delta_{R(L)}^{2}\right)^{1 / 2}$ and $K=1+2 Z^{2}$. From Eq. (16) we obtain

$$
I=-\frac{2 e}{\hbar \beta} \sum_{\omega_{n}} \frac{\Delta_{R} \Delta_{L} \sin \varphi}{\omega_{n}^{2}+K \Omega_{n R} \Omega_{n L}+\Delta_{R} \Delta_{L} \cos \varphi}
$$

where $\Omega_{n R(L)}=\left(\omega_{n}^{2}+\Delta_{R(L)}^{2}\right)^{1 / 2}$. This result agrees with the Ambegaokar-Baratoff formula [25] for a tunnel junction in the limit $K \gg 1$ and with the Kulik-Omel'yanchuk formula [11] for a point contact with perfect transmission in the limit $K \rightarrow 1$. The summation can be reduced to an integral over $\Delta_{L}<|E|<\Delta_{R}$ plus contributions from residues at energies of bound states at $|E|<\Delta_{L}$. The integral over $\Delta<|E|<\Delta_{R}$ corresponds to the process shown in Fig. $\theta$ and carry most of the supercurrent. 


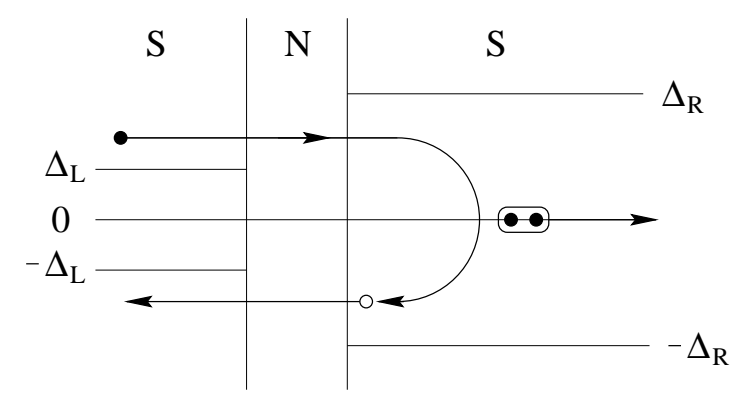

FIG. 4. Scattering states carrying supercurrent in a short asymmetric junction.

\section{QUANTIZATION OF SUPERCURRENT}

In a superconducting quantum point contact, the critical current or the maximum value of the dc Josephson current can take a quantized value in units of $e \Delta_{0} / \hbar$. This was first predicted theoretically [1,2] and confirmed by experiments [3. 1 . The effect can be understood easily by the following argument. The critical current of a Josephson junction is generally proportional to its normal-state conductance, as seen in Eq. (11b). It is well known that the conductance of a quantum point contact fabricated in a 2DEG is quantized in units of $2 e^{2} / h$ 38, 39]: The conductance is simply $2 e^{2} / h$ times the number of open channels at the constriction. We can thus naively expect that the critical current should be quantized as well. For a more quantitative discussion we can use the adiabatic approximation where scattering between channels is neglected. The transmission probability through the quantum point contact is then $\mathcal{T}_{j}=1$ for open channels and $\mathcal{T}_{j}=0$ for closed channels. For a short symmetric superconducting quantum point contact where $\hbar v_{F} / L \gg \Delta_{0}$, we obtain, from Eqs. (11b) and (16),

$$
I=-N \frac{e \Delta_{0}}{\hbar} \sin (\varphi / 2) \tanh \left(\frac{\Delta_{0}}{2 k_{B} T} \cos (\varphi / 2)\right)
$$

where $N$ is the number of open channels at the point contact. We thus find that the critical current is a multiple of $e \Delta_{0} / \hbar$ at zero temperature. The number of open channels is determined by the width of the constriction, which can be controlled externally, for example, by changing a gate voltage for a 2DEG. This was done in an experiment by Takayanagi et al. [3].

Equation (21) is valid only for short junctions. In the opposite case where $\hbar v_{F} / L \ll \Delta_{0}$, the unit of the quantization is different. The supercurrent for a long superconducting quantum point contact is given by

$$
I \approx-\frac{e}{\pi L} \sum_{j=1}^{N} v_{j} \varphi
$$

for $|\varphi|<\pi$ at zero temperature, where $v_{j}$ is the longitudinal component of the Fermi velocity in $j$ th channel. In this case the critical current is not exactly quantized in contrast with the short-junction limit. This is nothing but a quantum limit of long SNS junctions obtained in early 1970s [7, 8 .

\section{CONCLUSIONS}

In this paper we have reviewed the dc Josephson effect in mesoscopic Josephson junctions. Our discussion is based on a simple analysis of the Bogoliubov-de Gennes equation. In short junctions including superconducting quantum point contacts the whole supercurrent is carried by Andreev bound states in the energy gap that appear as a result of multiple Andreev reflections. The wave function of a bound state is constructed explicitly for a simple $\delta$-function model by solving the Bogoliubov-de Gennes equation. The bound state energies are sensitive to the difference of the phases of superconductors, and this sensitivity is the origin of the Josephson effect. Our simple physical view of the Josephson effect in terms of the current-carrying bound states is very general and applicable to many situations. 


\section{ACKNOWLEDGMENTS}

The author thanks M. Sigrist and A. M. Zagoskin for helpful comments on the manuscript.

* Permanent address.

[1] A. Furusaki, H. Takayanagi and M. Tsukada, Phys. Rev. Lett. 67, 132 (1991); Phys. Rev. B 45, 10563 (1992).

[2] C.W.J. Beenakker and H. van Houten, Phys. Rev. Lett. 66, 3056 (1991).

[3] H. Takayanagi, J.B. Hansen and J. Nitta, Phys. Rev. Lett. 74, 166 (1995); H. Takayanagi, T. Akazaki and J. Nitta, Phys. Rev. Lett. 75, 3533 (1995).

[4] C.J. Muller, J.M. van Ruitenbeek, and L.J. de Jongh, Phys. Rev. Lett. 69, 140 (1992).

[5] A.F. Andreev, Sov. Phys. JETP 19, 1228 (1964).

[6] I.O. Kulik, Sov. Phys. JETP 30, 944 (1970).

[7] C. Ishii, Prog. Theor. Phys. 44, 1525 (1970); 47, 1464 (1972).

[8] J. Bardeen and J.L. Johnson, Phys. Rev. B 5, 72 (1972).

[9] A.V. Svidzinsky, T.N. Antsygina and E.N. Bratus', J. Low Temp. Phys. 10, 131 (1973).

[10] A. Furusaki and M. Tsukada, Physica B165\&166, 967 (1990); A. Furusaki and M. Tsukada, Phys. Rev. B 43, 10164 (1991).

[11] I.O. Kulik and A.N. Omel'yanchuk, Sov. J. Low Temp. Phys. 4, 142 (1978).

[12] A.V. Zaitsev, Sov. Phys. JETP 59, 1015 (1984).

[13] G.B. Arnold, J. Low Temp. Phys. 59, 143 (1985).

[14] T.M. Klapwijk, G.E. Blonder, and M. Tinkham, Physica B\&C 110, 1657 (1982).

[15] S.N. Artemenko, A.F. Volkov and A.V. Zaitsev, Sov. Phys. JETP 49, 924 (1979).

[16] K. Flensberg, J.B. Hansen, and M. Octavio, Phys. Rev. B 38, 8707 (1988).

[17] R. Kümmel, U. Gunsenheimer and P. Nicolsky, Phys. Rev. B 42, 3992 (1990).

[18] U. Gunsenheimer and A.D. Zaikin, Phys. Rev. B 50, 6317 (1995).

[19] E.N. Bratus', V.S. Shumeiko and G. Wendin, Phys. Rev. Lett. 74, 2110 (1995).

[20] D. Averin and A. Bardas, Phys. Rev. Lett. 75, 1831 (1995); Phys. Rev. B 53, R1705 (1996); D. Averin and H.T. Imam, Phys. Rev. Lett. 76, 3814 (1996).

[21] M. Hurd, S. Datta, and P.F. Bagwell, Phys. Rev. B 54, 6557 (1996).

[22] P.G. de Gennes, Superconductivity of Metals and Alloys (Benjamin, New York, 1966).

[23] G.E. Blonder, M. Tinkham and T.M. Klapwijk, Phys. Rev. B 25, 4515 (1982).

[24] A. Furusaki and M. Tsukada, Solid State Commun. 78, 299 (1991).

[25] V. Ambegaokar and A. Baratoff, Phys. Rev. Lett. 10, 486 (1963); 11, 104(E) (1964).

[26] W. Haberkorn, H. Knauer, and J. Richter, Phys. Stat. Sol. (A) 47, K161 (1978).

[27] L.I. Glazman and K.A. Matveev, JETP Lett. 49, 659 (1989).

[28] C.W.J. Beenakker, Phys. Rev. Lett. 67, 3836 (1991); 68, 1442(E) (1992).

[29] C. Caroli, P.G. de Gennes, and J. Matricon, Phys. Lett. 6, 307 (1964).

[30] C.R. Hu, Phys. Rev. Lett. 72, 1526 (1994).

[31] Y. Tanaka and S. Kashiwaya, Phys. Rev. Lett. 74, 3451 (1995).

[32] S. Yip, J. Low Temp. Phys. 91, 203 (1993); Y. Tanaka, Phys. Rev. Lett. 72, 3871 (1994); A. M. Zagoskin, J. Phys.: Condens. Matter 9, L419 (1997).

[33] A. M. Zagoskin, Quantum Theory of Many-Body Systems: Techniques and Applications (Springer-Verlag, New York, 1998).

[34] See, for example, M. Sigrist, Prog. Theor. Phys. 99, 899 (1998) and references therein.

[35] K.K. Likharev, Rev. Mod. Phys. 51, 101 (1979).

[36] A.A. Abrikosov, L.P. Gorkov and I.E. Dzyaloshinski, Methods of Quantum Field Theory in Statistical Physics (Dover, New York, 1975).

[37] A complementary method to compute the dc Josephson current in lattice models is found in A. Furusaki, Physica B203, 214 (1994). There is a small but important typo in this paper: The left hand side of Eq. (10) should read $G_{\omega_{n}}\left(j, k ; j, k^{\prime}\right)$. See also Y. Takane, J. Phys. Soc. Jpn. 63, 2668 (1994); Y. Koyama, Y. Takane and H. Ebisawa, J. Phys. Soc. Jpn. 66, 430 (1997); 66, 3314(E) (1997).

[38] B.J. van Wees, H. van Houten, C.W.J. Beenakker, J.G. Williamson, L.P. Kouwenhoven, D. van der Marel and C.T. Foxon, Phys. Rev. Lett. 60, 848 (1988).

[39] D.A. Wharam, T.J. Thornton, R. Newbury, M. Pepper, H. Ahmed, J.E.F. Frost, D.G. Hasko, D.C. Peacock, D.A. Ritchie and G.A.C. Jones, J. Phys. C21, L209 (1988). 